\title{
Global Attractor for Partial Functional Differential Equations with State-Dependent Delay
}

\author{
Zhimin $\mathrm{He}^{1}$ and $\mathrm{Bo} \mathrm{Du}^{2}$ \\ ${ }^{1}$ College of Science, Zhejiang A and F University, Hangzhou, Zhejiang 311300, China \\ ${ }^{2}$ Department of Mathematics, Huaiyin Normal University, Huaian, Jiangsu 223300, China
}

Correspondence should be addressed to Bo Du; dubo7307@163.com

Received 25 March 2013; Accepted 28 June 2013

Academic Editor: Ferenc Hartung

Copyright (C) 2013 Z. He and B. Du. This is an open access article distributed under the Creative Commons Attribution License, which permits unrestricted use, distribution, and reproduction in any medium, provided the original work is properly cited.

\begin{abstract}
This work aims to investigate the existence of global attractors for a class of partial functional differential equations with statedependent delay. Using the classic theory about global attractors in infinite dimensional dynamical systems, we obtain some sufficient conditions for guaranteeing the existence of a global attractor.
\end{abstract}

\section{Introduction}

Partial functional differential equations with state-dependent delay appear frequently in applications as models of various phenomena, such as biological, chemical, and physical systems, which are characterized by both spatial and temporal variables. For this reason, the study of this kind of equation has received much attention in recent years. For more details, see for instance [1-5] and the references therein. However, it is worth pointing out that all of the papers mentioned above are mainly devoted to the existence of solutions or mild solutions. The literature related to global attractors is limited.

It is known that the global attractor is a very useful tool, which is valid for more general situations than those for stability to study the asymptotical behavior. In the present paper, we are devoted to investigating the existence of a global attractor for a type of partial functional differential equations with state-dependent delay as follows:

$$
\begin{gathered}
u^{\prime}(t)=A u(t)+F\left(t, u_{\rho\left(t, u_{t}\right)}\right), \quad t \geq 0, \\
u_{0}=\phi \in C,
\end{gathered}
$$

where $C:=C([-r, 0], E), r>0$, is the space of continuous functions from $[-r, 0]$ to the Banach space $E$, equipped with the uniform norm $\|\phi\|=\sup _{-r \leq \theta \leq 0}|\phi(\theta)|$, and $A$ is a linear operator on a Banach space $E$ satisfying the following wellknown Hille-Yosida condition:

$\left(\mathrm{H}_{1}\right)$ there exist $M_{0} \geq 1$ and $\omega \in \mathbb{R}$ such that

$$
\left\|(\lambda I-A)^{-n}\right\| \leq \frac{M_{0}}{(\lambda-\omega)^{n}} \quad \text { for } \lambda \in \mathscr{R}(A), \lambda>\omega,
$$

where $\mathscr{R}(A)$ is the resolvent set of $A$.

Consider that $\rho:[0,+\infty) \times C \rightarrow[0,+\infty)$ satisfies the following properties.

$\left(\mathrm{H}_{2}\right)$ Let $\mathscr{L}(\rho)=\{\rho(s, \phi):(s, \phi) \in[0,+\infty) \times C, \rho(s, \phi) \geq$ $0\}$. There exists a continuous and bounded function $J^{\phi}: \mathscr{L}(\rho) \rightarrow[0,+\infty)$ such that

$$
\left\|\varphi_{\rho\left(t, x_{t}\right)}\right\| \leq J^{\phi}(t)\left\|\phi_{t}\right\|, \quad \forall t \in \mathscr{L}(\rho) .
$$

Consider that $F:[0,+\infty) \times C \rightarrow E$ satisfies the following properties.

$\left(\mathrm{H}_{3}\right)$ (i) For every $\phi \in C$, the function $t \rightarrow F(t, \phi)$ is strongly measurable.

(ii) For each $t \in[0,+\infty], \quad F(t, \cdot): C \rightarrow E$ is continuous. 
(iii) There exist a positive constant $c$ and a bounded function $m:[0,+\infty) \rightarrow[0,+\infty)$ such that

$$
|F(t, \phi)| \leq c+m(t)\|\phi\| \text {. }
$$

For every $t \geq 0$, the history function $x_{t} \in C$ is defined by

$$
x_{t}(\theta)=x(t+\theta), \quad \text { for } \theta \in[-r, 0] .
$$

In the present paper, we will obtain some sufficient conditions for guaranteeing the existence of a global attractor to (1) with $A$ being a Hille-Yosida operator but not necessarily densely defined.

\section{Preliminaries}

We recall some definitions and results from the integrated semigroup.

Definition 1 (see [6]). Let $T>0$. A function $x:[-r, T] \rightarrow E$ is said to be an integral solution of (1) if

(i) $\int_{0}^{t} x(s) d s \in D(A)$ for $t \geq 0$,

(ii) $x(t)=\phi(0)+A\left(\int_{0}^{t} x(s) d s\right)+\int_{0}^{t} F\left(s, x_{\rho\left(s, x_{s}\right)}\right) d s$,

(iii) $x_{0}=\phi$.

Remark 2. Clearly, if $x$ is an integral solution of (1), then $x_{t}(0)=x(t) \in \overline{D(A)}$ for $t \in[0, T]$. So $\phi(0) \in \overline{D(A)}$, which is a necessary condition for the existence of an integral solution.

Let us introduce the part $A_{0}$ of the operator $A$ in $\overline{D(A)}$ which is defined by

$$
\begin{gathered}
D\left(A_{0}\right)=\{x \in D(A): A x \in \overline{D(A)}\}, \\
A_{0} x=A x \quad \text { for } x \in D\left(A_{0}\right) .
\end{gathered}
$$

Lemma 3 (see [7]). A generates a strongly continuous semigroup $\left(T_{0}(t)\right)_{t \geq 0}$ on $\overline{D(A)}$.

Based on the previous abstract results, we give some concrete results for (1); see [6].

Definition 4. Let $T>0$. For any given $\phi \in C$ with $\phi(0) \in$ $\overline{D(A)}$, the function $x(\cdot):=x(\cdot, \phi):[-r, T] \rightarrow E$ is said to be an integral solution of (1) with initial function $\phi$ at $t=0$ if

$x(t)$

$$
= \begin{cases}T_{0}(t) \phi(0) & \\ \quad+\lim _{\lambda \rightarrow+\infty} \int_{0}^{t} T_{0}(t-s) & \\ \quad \times \lambda(\lambda I-A)^{-1} F\left(s, x_{\rho\left(s, x_{s}\right)}\right) d s, & 0 \leq t \leq T, \\ \phi(t), & -r \leq t \leq 0 .\end{cases}
$$

Lemma 5 (see [6]). Under the assumptions $\left(H_{1}\right)-\left(H_{3}\right)$, if $\phi \in$ $C$ with $\phi(0) \in \overline{D(A)}$, then (1) posseses a unique global integral solution $x(\cdot, \phi):[-r,+\infty) \rightarrow E$ with initial function $\phi$ at $t=0$, which can be expressed by (7).
According to Remark 2, denote $\Sigma_{0}=\{\phi \in C: \phi(0) \epsilon$ $\overline{D(A)}\}$. Then from Lemma 5 , for each $\phi \in \Sigma_{0}$, we define the following operator on $\Sigma_{0}$ by

$$
U(t) \phi=x_{t}(\cdot, \phi), \quad t \geq 0,
$$

where $x_{t}(\cdot, \phi)$ is a unique global integral solution of (1) in Lemma 5. Clearly, $(U(t))_{t \geq 0}$ is a strongly continuous semigroup on $\Sigma_{0}$.

Definition 6 (see [8]). An invariant set $\mathscr{A}$ is said to be a global attractor if $\mathscr{A}$ is a maximal compact invariant set which attracts each bounded set $B \subset X$.

Definition 7 (see [8]). A semigroup $U(t): X \rightarrow X, t \geq 0$, is said to be point dissipative if there is a bounded set $B \subseteq X$ that attracts each point of $E$ under $U(t)$.

Lemma 8 (see [9]). If

(i) there is a $t_{0} \geq 0$ such that $U(t)$ is compact for $t>t_{0}$,

(ii) $U(t)$ is point dissipative in $X$,

then there exists a nonempty global attractor $\mathscr{A}$ in $X$.

\section{The Global Attractor}

In this section, we will obtain the existence of a global attractor to (7) by using Lemma 8. For the convenience of the proof, we give some assumptions and lemmas.

$\left(\mathrm{H}_{4}\right)$ For $C_{0}$-semigroup $T_{0}(t), t \geq 0$, there exists positive constant $\alpha$ such that

$$
\left\|T_{0}(t)\right\| \leq e^{-\alpha t} \quad \text { for } t \geq 0 .
$$

$\left(\mathrm{H}_{5}\right) T_{0}(t)$ is compact for $t>0$.

Lemma 9 (see [10]). If

$$
x(t) \leq h(t)+\int_{t_{0}}^{t} k(s) x(s) d s, \quad t \in\left[t_{0}, T\right),
$$

where all the functions involved are continuous on $\left[t_{0}, T\right), T \leq$ $+\infty$, and $k(t) \geq 0$, then $x(t)$ satisfies

$$
x(t) \leq h(t)+\int_{t_{0}}^{t} h(s) k(s) e^{\int_{s}^{t} k(u) d u} d s, \quad t \in\left[t_{0}, T\right) .
$$

Lemma 10. Assume that assumptions $\left(H_{1}\right)-\left(H_{4}\right)$ hold. Then, for each $\phi \in \Sigma_{0}$, if $\alpha \neq m_{a} J_{a} e^{\gamma r}$, there exists a constant $\gamma>$ $\alpha$ such that the integral solution $x(\cdot, \phi)$ of (1) satisfies the following inequality:

$$
\begin{aligned}
\left\|x_{t}\right\| \leq & \frac{c e^{\gamma r}}{\alpha-m_{a} J_{a} e^{\gamma r}} \\
& +e^{\gamma r}\left(\|\phi\|-\frac{c}{\alpha-m_{a} J_{a} e^{\gamma r}}\right) e^{\left(m_{a} J_{a} e^{\gamma r}-\alpha\right) t}, \quad t \geq 0,
\end{aligned}
$$

where $m_{a}=\max _{s \in[0, \infty)} m(s)$ and $J_{a}=\max _{s \in \mathscr{L}(\rho)} J^{\phi}(s)$. 
Proof. By $\left(\mathrm{H}_{3}\right)$, for each $\phi \in \Sigma_{0}$, we have

$$
|F(t, \phi)| \leq c+m(t)\|\phi\| \text {. }
$$

In the following proof, for simplicity, take $M_{0}=1, n=1$ in $\left(\mathrm{H}_{1}\right)$; then

$$
\left\|(\lambda I-A)^{-1}\right\| \leq \frac{1}{\lambda-\omega} \text { for } \lambda>\omega .
$$

Instead of considering the norm $\left\|x_{t}\right\|$ directly, we firstly estimate $\left\|e^{\gamma} x_{t}\right\|$ for some constant $\gamma>\alpha$.

Case 1 . For $0 \leq t \leq r$, by (7), we have

$$
\begin{aligned}
& \sup _{-r \leq \theta \leq 0}\left|e^{\gamma \theta} x_{t}(\theta)\right| \\
& =\max \left\{\sup _{-r \leq \theta \leq-t}\left|e^{\gamma \theta} \phi(t+\theta)\right|, \sup _{-t \leq \theta \leq 0}\left|e^{\gamma \theta} x_{t}(\theta)\right|\right\} \\
& \leq \max \left\{e^{-\gamma t}\|\phi\|, \sup _{-t \leq \theta \leq 0} e^{\gamma \theta} e^{-\alpha(t+\theta)}|\phi(0)|\right. \\
& +\sup _{-t \leq \theta \leq 0} e^{\gamma \theta} \lim _{\lambda \rightarrow+\infty} \int_{0}^{t+\theta} e^{-\alpha(t+\theta-s)}\left\|\lambda(\lambda I-A)^{-1}\right\| \\
& \left.\times\left[c+m(s) J^{\phi}(s)\left\|x_{s}\right\|\right] d s\right\} \\
& \leq \max \left\{e^{-\gamma t}\|\phi\|, e^{-\alpha t}|\phi(0)|\right. \\
& +\sup _{-t \leq \theta \leq 0} c e^{-\alpha(t+\theta)} e^{\gamma \theta} \lim _{\lambda \rightarrow+\infty} \int_{0}^{t+\theta} \frac{\lambda}{\lambda-\omega} e^{\alpha s} d s \\
& +\sup _{-t \leq \theta \leq 0} m_{a} J_{a} e^{-\alpha(t+\theta)} e^{\gamma \theta} \\
& \left.\times \lim _{\lambda \rightarrow+\infty} \int_{0}^{t+\theta} \frac{\lambda}{\lambda-\omega} e^{\alpha s}\left\|x_{s}\right\| d s\right\} \\
& \leq e^{-\alpha t}\|\phi\|+c e^{-\alpha t} \int_{0}^{t} e^{\alpha s} d s+m_{a} J_{a} e^{-\alpha t} \int_{0}^{t} e^{\alpha s}\left\|x_{s}\right\| d s \\
& =e^{-\alpha t}\|\phi\|+\frac{c}{\alpha}\left(1-e^{-\alpha t}\right)+m_{a} J_{a} e^{-\alpha t} \int_{0}^{t} e^{\alpha s}\left\|x_{s}\right\| d s .
\end{aligned}
$$

Case 2. For $t \geq r$, we have

$$
\begin{aligned}
& \sup _{-r \leq \theta \leq 0}\left|e^{\gamma \theta} x_{t}(\theta)\right| \\
& \leq \sup _{-r \leq \theta \leq 0} e^{\gamma \theta} e^{-\alpha(t+\theta)}|\phi(0)| \\
& \quad+\sup _{-r \leq \theta \leq 0} e^{\gamma \theta} \lim _{\lambda \rightarrow+\infty} \int_{0}^{t+\theta} e^{-\alpha(t+\theta-s)}\left\|\lambda(\lambda I-A)^{-1}\right\| \\
& \quad \times\left[c+m_{a} J_{a}\left\|x_{s}\right\|\right] d s
\end{aligned}
$$

$$
\begin{aligned}
\leq & e^{-\alpha t}\|\phi\|+\frac{c}{\alpha}\left(1-e^{-\alpha t}\right) \\
& +m_{a} J_{a} e^{-\alpha t} \int_{0}^{t} e^{\alpha s}\left\|x_{s}\right\| d s .
\end{aligned}
$$

Therefore, from (15) and (16), for $t \geq 0$, we get

$$
\begin{aligned}
\sup _{-r \leq \theta \leq 0}\left|e^{\gamma \theta} x_{t}(\theta)\right| \leq & e^{-\alpha t}\|\phi\|+\frac{c}{\alpha}\left(1-e^{-\alpha t}\right) \\
& +m_{a} J_{a} e^{-\alpha t} \int_{0}^{t} e^{\alpha s}\left\|x_{s}\right\| d s .
\end{aligned}
$$

On the other hand, we have

$$
\begin{aligned}
\sup _{-r \leq \theta \leq 0}\left|e^{\gamma \theta} x_{t}(\theta)\right| & =\sup _{-r \leq \theta \leq 0} e^{\gamma \theta}\left|x_{t}(\theta)\right| \\
& \geq \sup _{-r \leq \theta \leq 0} e^{-\gamma r}\left|x_{t}(\theta)\right| \\
& =e^{-\gamma r}\left\|x_{t}\right\|,
\end{aligned}
$$

which combines with (17) and yields

$$
e^{-\gamma r}\left\|x_{t}\right\| \leq e^{-\alpha t}\|\phi\|+\frac{c}{\alpha}\left(1-e^{-\alpha t}\right)+m_{a} J_{a} e^{-\alpha t} \int_{0}^{t} e^{\alpha s}\left\|x_{s}\right\| d s .
$$

So we get

$$
e^{\alpha t}\left\|x_{t}\right\| \leq e^{\gamma r}\left[\|\phi\|+\frac{c}{\alpha}\left(e^{\alpha t}-1\right)\right]+m_{a} J_{a} e^{\gamma r} \int_{0}^{t} e^{\alpha s}\left\|x_{s}\right\| d s .
$$

Using Lemma 9, we have

$$
\begin{aligned}
e^{\alpha t}\left\|x_{t}\right\| \leq & e^{\gamma r}\|\phi\|+\frac{c}{\alpha} e^{\gamma r}\left(e^{\alpha t}-1\right) \\
& +m_{a} J_{a} e^{\gamma r} \int_{0}^{t}\left[e^{\gamma r}\|\phi\|+\frac{c}{\alpha} e^{\gamma r}\left(e^{\alpha s}-1\right)\right] \\
& \times e^{\int_{s}^{t} m_{a} J_{a} e^{\gamma r} d u} d s \\
\leq & \frac{c e^{\gamma r} e^{\alpha t}}{\alpha-m_{a} J_{a} e^{\gamma r}}+e^{\gamma r}\left(\|\phi\|-\frac{c}{\alpha-m_{a} J_{a} e^{\gamma r}}\right) e^{m_{a} J_{a} e^{\gamma r} t} .
\end{aligned}
$$

Thus, (12) holds.

Lemma 11. Assume that the conditions of Lemma 10 are satisfied; furthermore, $\alpha>m_{a} J_{a} e^{\gamma r}$, where $\gamma$ is the constant defined by Lemma 10. Then $(U(t))_{t \geq 0}$ is point dissipative.

Proof. From Lemma 10, we find that for each $\phi \in \Sigma_{0}$, since $\alpha>m_{a} J_{a} e^{\gamma r}$, there exits a $t_{0}:=t_{0}(\phi)>0$ such that for $t>t_{0}$,

$$
\left.\left\|x_{t}\right\| \leq \frac{c e^{\gamma r}}{\alpha-m_{a} J_{a} e^{\gamma r}}+1 \quad \text { (independent of } \phi\right) .
$$


Therefore,

$$
B_{X_{0}}\left(0, \frac{c e^{\gamma r}}{\alpha-m_{a} J_{a} e^{\gamma r}}+1\right) \cap X_{0}
$$

attracts each point of $X_{0}$, where $B_{X_{0}}\left(0,\left(c e^{\gamma r} /\left(\alpha-m_{a} J_{a} e^{\gamma r}\right)\right)+\right.$ 1) denotes the open ball in $\Sigma_{0}$ with center 0 and radius $\left(c e^{\gamma r} /\left(\alpha-m_{a} J_{a} e^{\gamma r}\right)\right)+1$.

Now, we show the compactness of the operator $U(t)$.

Lemma 12. Assume that assumptions $\left(H_{1}\right)-\left(H_{5}\right)$ hold. Then, $U(t)$ is compact for $t>r$.

Proof. Let $t>r$ and let $\left\{\phi_{n}\right\}$ be any bounded sequence of $\Sigma_{0}$. We will use the Ascoli-Arzelà theorem to show that $\left\{U(t) \phi_{n}\right.$ : $n \in \mathbb{N}\}$ is precompact in $\Sigma_{0}$ by two steps.

Step 1. Show that for any $\theta \in[-r, 0]$, the set

$$
Z(\theta)=\left\{\left(\left(U(t) \phi_{n}\right)(\theta): n \in \mathbb{N}\right)\right\}
$$

is precompact. For $t>r$ and $\theta \in[-r, 0]$, by (8), we have

$$
\begin{aligned}
&\left(U(t) \phi_{n}\right)(\theta)= T_{0}(t+\theta) \phi_{n}(0) \\
&+\lim _{\lambda \rightarrow+\infty} \int_{0}^{t+\theta} T_{0}(t+\theta-s) \\
& \quad \times \lambda(\lambda I-A)^{-1} F\left(x_{\rho\left(s, x_{s}\right)}^{n}\right) d s,
\end{aligned}
$$

where $x^{n}(\cdot)$ is the integer solution of (1) with initial function $\phi_{n}$. From $\left(\mathrm{H}_{4}\right)$ and the boundedness of $\left\{\phi_{n}\right\}$, we know that $\left\{T_{0}(t+\theta) \phi_{n}(0): n \in \mathbb{N}\right\}$ are precompact. Now, considering the second term in (25), for sufficiently small $\varepsilon>0$, we have

$$
\begin{aligned}
& \lim _{\lambda \rightarrow+\infty} \int_{0}^{t+\theta} T_{0}(t+\theta-s) \lambda(\lambda I-A)^{-1} F\left(x_{\rho\left(s, x_{s}\right)}^{n}\right) d s \\
& =T_{0}(\varepsilon) \lim _{\lambda \rightarrow+\infty} \int_{0}^{t+\theta-\varepsilon} T_{0}(t+\theta-s-\varepsilon) \lambda(\lambda I-A)^{-1} \\
& \quad \times F\left(x_{\rho\left(s, x_{s}\right)}^{n}\right) d s \\
& \quad+\lim _{\lambda \rightarrow+\infty} \int_{t+\theta-\varepsilon}^{t+\theta} T_{0}(t+\theta-s) \lambda(\lambda I-A)^{-1} F\left(x_{\rho\left(s, x_{s}\right)}^{n}\right) d s .
\end{aligned}
$$

Note that from Lemma 10, we have

$$
\sup _{n \in \mathbb{N}}\left\|x_{s}^{n}\right\|<\infty, \quad s \in[0, t] .
$$

By $\left(\mathrm{H}_{2}\right)$ and $\left(\mathrm{H}_{3}\right)$, we get

$$
\left|F\left(x_{\rho\left(s, x_{s}\right)}^{n}\right)\right| \leq c+m_{a} J_{a}\left\|x_{s}^{n}\right\| .
$$

Therefore, there exist some constants $M_{1}, M_{2}>0$ such that

$$
\begin{aligned}
& \left|\lim _{\lambda \rightarrow+\infty} \int_{0}^{t+\theta-\varepsilon} T_{0}(t+\theta-s-\varepsilon) \lambda(\lambda I-A)^{-1} F\left(x_{\rho\left(s, x_{s}\right)}^{n}\right) d s\right| \\
& \quad \leq M_{1} \\
& \quad\left|\lim _{\lambda \rightarrow+\infty} \int_{t+\theta-\varepsilon}^{t+\theta} T_{0}(t+\theta-s) \lambda(\lambda I-A)^{-1} F\left(x_{\rho\left(s, x_{s}\right)}^{n}\right) d s\right| \\
& \quad \leq M_{2} \varepsilon
\end{aligned}
$$

which yields

$$
\begin{aligned}
T_{0}(\varepsilon)\left\{\lim _{\lambda \rightarrow+\infty} \int_{0}^{t+\theta-\varepsilon} T_{0}(t+\theta-s-\varepsilon)\right. \\
\left.\quad \times \lambda(\lambda I-A)^{-1} F\left(x_{\rho\left(s, x_{s}\right)}^{n}\right) d s: n \in \mathbb{N}\right\} \subset \Gamma_{\varepsilon},
\end{aligned}
$$

where $\Gamma_{\varepsilon}$ is a compact set. Thus, $Z(\theta)$ is precompact.

Step 2. Show the equicontinuity of $\left\{U(t) \phi_{n}: n \in \mathbb{N}\right\}$. Let $-r \leq$ $\theta_{1}<\theta_{2} \leq 0$; we have

$$
\begin{aligned}
(U(t) & \left.\phi_{n}\right)\left(\theta_{2}\right)-\left(U(t) \phi_{n}\right)\left(\theta_{1}\right) \\
= & {\left[T_{0}\left(t+\theta_{2}\right)-T_{0}\left(t+\theta_{1}\right)\right] \phi_{n}(0) } \\
& +\lim _{\lambda \rightarrow+\infty} \int_{0}^{t+\theta_{2}} T_{0}\left(t+\theta_{2}-s\right) \lambda(\lambda I-A)^{-1} F\left(x_{\rho\left(s, x_{s}\right)}^{n}\right) d s \\
& -\lim _{\lambda \rightarrow+\infty} \int_{0}^{t+\theta_{1}} T_{0}\left(t+\theta_{1}-s\right) \lambda(\lambda I-A)^{-1} F\left(x_{\rho\left(s, x_{s}\right)}^{n}\right) d s \\
= & T_{0}\left(t+\theta_{1}\right)\left[T_{0}\left(\theta_{2}-\theta_{1}\right)-I\right] \phi_{n}(0) \\
& +\lim _{\lambda \rightarrow+\infty} \int_{t+\theta_{1}}^{t+\theta_{2}} T_{0}\left(t+\theta_{2}-s\right) \lambda(\lambda I-A)^{-1} F\left(x_{\rho\left(s, x_{s}\right)}^{n}\right) d s \\
& +\lim _{\lambda \rightarrow+\infty} \int_{0}^{t+\theta_{1}}\left[T_{0}\left(t+\theta_{2}-s\right)-T_{0}\left(t+\theta_{1}-s\right)\right] \\
& \quad \times \lambda(\lambda I-A)^{-1} F\left(x_{\rho\left(s, x_{s}\right)}^{n}\right) d s,
\end{aligned}
$$

which leads to

$$
\begin{aligned}
& \left|\left(U(t) \phi_{n}\right)\left(\theta_{2}\right)-\left(U(t) \phi_{n}\right)\left(\theta_{1}\right)\right| \\
& \leq\left\|T_{0}\left(t+\theta_{1}\right)\left[T_{0}\left(\theta_{2}-\theta_{1}\right)-I\right]\right\| \\
& +\lim _{\lambda \rightarrow+\infty} \int_{t+\theta_{1}}^{t+\theta_{2}} \mid T_{0}\left(t+\theta_{2}-s\right) \lambda(\lambda I-A)^{-1} \\
& \quad \times F\left(x_{\rho\left(s, x_{s}\right)}^{n}\right) \mid d s+\left\|T_{0}\left(\theta_{2}-\theta_{1}\right)-I\right\|
\end{aligned}
$$




$$
\begin{gathered}
\times \lim _{\lambda \rightarrow+\infty} \int_{0}^{t+\theta_{1}} \mid T_{0}\left(t+\theta_{1}-s\right) \lambda(\lambda I-A)^{-1} \\
\times F\left(x_{\rho\left(s, x_{s}\right)}^{n}\right) \mid d s .
\end{gathered}
$$

Since the mapping $t \rightarrow T_{0}(t)$ is norm continuous for $t>0$, for some $\delta \in(0, t-r)$, put

$$
\begin{aligned}
T_{0}(t & \left.+\theta_{1}\right)\left[T_{0}\left(\theta_{2}-\theta_{1}\right)-I\right] \\
& =T_{0}\left(t+\theta_{1}-\delta\right)\left[T_{0}\left(\theta_{2}-\theta_{1}+\delta\right)-T_{0}(\delta)\right] .
\end{aligned}
$$

Then

$$
\left\|T_{0}\left(\theta_{2}-\theta_{1}+\delta\right)-T_{0}(\delta)\right\| \longrightarrow 0 \quad \text { as } \theta_{2} \longrightarrow \theta_{1} .
$$

Thus

$$
\left\|T_{0}\left(t+\theta_{1}\right)\left[T_{0}\left(\theta_{2}-\theta_{1}\right)-I\right]\right\| \longrightarrow 0 \quad \text { as } \theta_{2} \longrightarrow \theta_{1}
$$

By the boundedness of $\left|T_{0}\left(t+\theta_{2}-s\right) \lambda(\lambda I-A)^{-1} F\left(x_{\rho\left(s, x_{s}\right)}^{n}\right)\right|$, then

$$
\begin{aligned}
\lim _{\lambda \rightarrow+\infty} \int_{t+\theta_{1}}^{t+\theta_{2}} \mid T_{0}\left(t+\theta_{2}-s\right) \lambda(\lambda I-A)^{-1} & \\
& \times F\left(x_{\rho\left(s, x_{s}\right)}^{n}\right) \mid d s \longrightarrow 0 \quad \text { as } \theta_{2} \longrightarrow \theta_{1} .
\end{aligned}
$$

Obviously, $\quad \lim _{\lambda \rightarrow+\infty} \int_{0}^{t+\theta_{1}} \mid T_{0}\left(t+\theta_{1}-s\right) \lambda(\lambda I-A)^{-1}$ $F\left(x_{\rho\left(s, x_{s}\right)}^{n}\right) \mid d s$ belongs to a compact subset of $E$; we have

$$
\begin{aligned}
&\left\|T_{0}\left(\theta_{2}-\theta_{1}\right)-I\right\| \\
& \times \lim _{\lambda \rightarrow+\infty} \int_{0}^{t+\theta_{1}} \mid T_{0}\left(t+\theta_{1}-s\right) \lambda(\lambda I-A)^{-1} \\
& \times F\left(x_{\rho\left(s, x_{s}\right)}^{n}\right) \mid d s \longrightarrow 0 \\
& \text { as } \theta_{2} \longrightarrow \theta_{1} .
\end{aligned}
$$

Hence $\left\{U(t) \phi_{n}: n \in \mathbb{N}\right\}$ is equicontinuity.

Here, we state our main theorem of this paper, which is an immediate consequence of Lemmas 8,11 , and 12 .

Theorem 13. Assume that assumptions $\left(H_{1}\right)-\left(H_{5}\right)$ hold. If $\alpha>$ $m_{a} J_{a} e^{\gamma r}$, then (1) has a nonempty global attractor $\mathscr{A}$.

As applications, we give the following example.

Let $E=C([0, \pi], \mathbb{R})$ and $C=C([-r, 0], E)$.

$$
\begin{aligned}
\frac{\partial}{\partial t} u(t, x)= & \frac{\partial^{2}}{\partial x^{2}} u(t, x)-\mu u(t, x) \\
& +f(u(t-r, x)), \quad 0 \leq x \leq \pi, t \geq 0, \\
u(t, 0)=u(t, \pi)=0, \quad t \geq 0, & \\
u(\theta, x)= & \phi(\theta) x, \quad 0 \leq x \leq \pi, \quad-r \leq \theta \leq 0,
\end{aligned}
$$

where $\mu>0$ is a constant, $u(t, \cdot) \in E$, and $f: C \rightarrow E$ satisfies $\left(\mathrm{H}_{3}\right)$. Define the operator $A$ by

$$
\begin{gathered}
D(A)=\left\{q \in C^{2}([0, \pi], \mathbb{R}): q(0)=q(\pi)=0\right\}, \\
A(q)=q^{\prime \prime}
\end{gathered}
$$

and $F: C \rightarrow E$ with

$$
F(\varphi)(x)=f(\varphi(-r)(x)), \quad x \in[0, \pi] .
$$

Then $A$ satisfies the Hille-Yosida condition in $E$ and

$$
\left\|(\lambda I-A)^{-1}\right\| \leq \frac{1}{\lambda} \quad \text { for } \lambda>0 .
$$

Moreover, the part $A_{0}$ of $A$ in $\overline{D(A)}$ is the infinitesimal generator of a strongly continuous semigroup $\left(T_{0}(t)\right)_{t \geq 0}$ on E such that

$$
\left\|T_{0}(t)\right\| \leq e^{-t} \quad \text { for } t \geq 0 .
$$

According to Theorem 13, if there exist $\gamma>\mu, m_{a}$ and $J_{a}$ such that $m_{a} J_{a} e^{\gamma r}<\mu$, then (38) has a global attractor.

\section{Acknowledgments}

This paper is supported by the Natural Science Foundation of Jiangsu Education Office (11KJB110002), Postdoctoral Foundation of Jiangsu (1102096C), and Postdoctoral Foundation of China (2012M511296).

\section{References}

[1] F. Hartung, T. Krisztin, H.-O. Walther, and J. Wu, "Functional differential equations with state-dependent delays: theory and applications," in Handbook of Differential Equations: Ordinary Differential Equations. Vol. III, pp. 435-545, Elsevier/NorthHolland, Amsterdam, The Netherlands, 2006.

[2] Y. Cao, J. Fan, and T. C. Gard, "The effects of state-dependent time delay on a stage-structured population growth model," Nonlinear Analysis: Theory, Methods \& Applications, vol. 19, no. 2, pp. 95-105, 1992.

[3] F. Hartung, "Linearized stability in periodic functional differential equations with state-dependent delays," Journal of Computational and Applied Mathematics, vol. 174, no. 2, pp. 201211, 2005.

[4] F. Hartung, "Parameter estimation by quasilinearization in functional differential equations with state-dependent delays: a numerical study," in Proceedings of the Third World Congress of Nonlinear Analysts, Part 7 (Catania, 2000), vol. 47, 2001, Nonlinear Analysis TMA 47 (7), 4557-4566, 2001.

[5] Y. Kuang and H. L. Smith, "Slowly oscillating periodic solutions of autonomous state-dependent delay equations," Nonlinear Analysis: Theory, Methods \& Applications, vol. 19, no. 9, pp. 855872, 1992.

[6] M. Adimy, K. Ezzinbi, and J. Wu, "Center manifold and stability in critical cases for for some partial functional differential equations," International Journal of Evolution Equations, vol. 2, pp. 69-95, 2006. 
[7] M. Adimy, A. Elazzouzi, and K. Ezzinbi, "Bohr-Neugebauer type theorem for some partial neutral functional differential equations," Nonlinear Analysis: Theory, Methods \& Applications, vol. 66, no. 5, pp. 1145-1160, 2007.

[8] J. K. Hale, Asymptotic Behavior of Dissipative Systems, vol. 25 of Mathematical Surveys and Monographs, American Mathematical Society, Providence, RI, USA, 1988.

[9] J. E. Billotti and J. P. LaSalle, "Dissipative periodic processes," Bulletin of the American Mathematical Society, vol. 77, pp. 10821088, 1971.

[10] C. Corduneanu, Principles of Differential and Integral Equations, Allyn and Bacon, Boston, Mass, USA, 1971. 


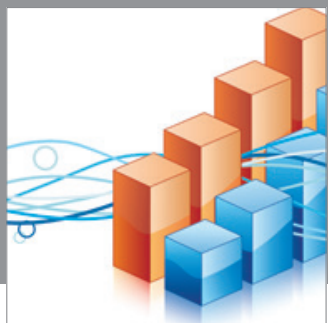

Advances in

Operations Research

mansans

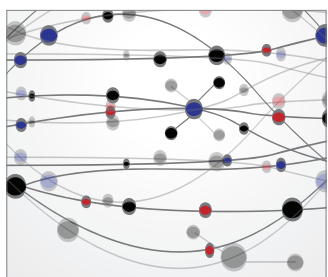

The Scientific World Journal
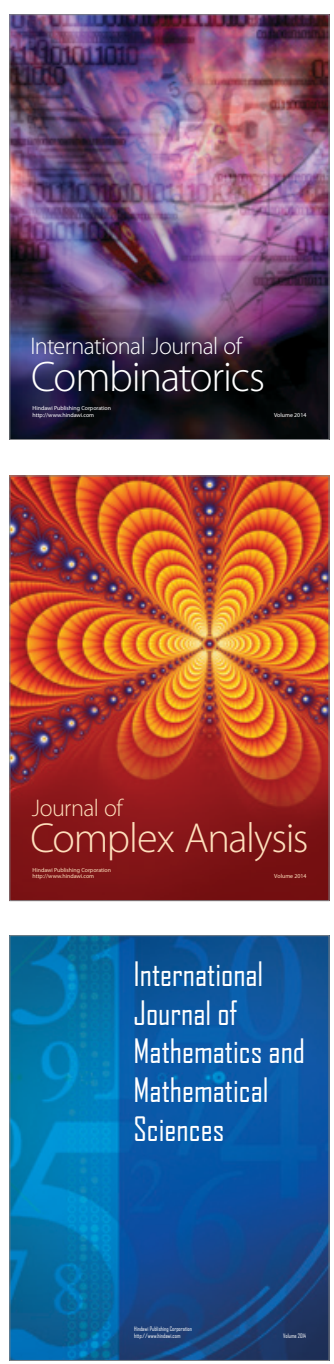
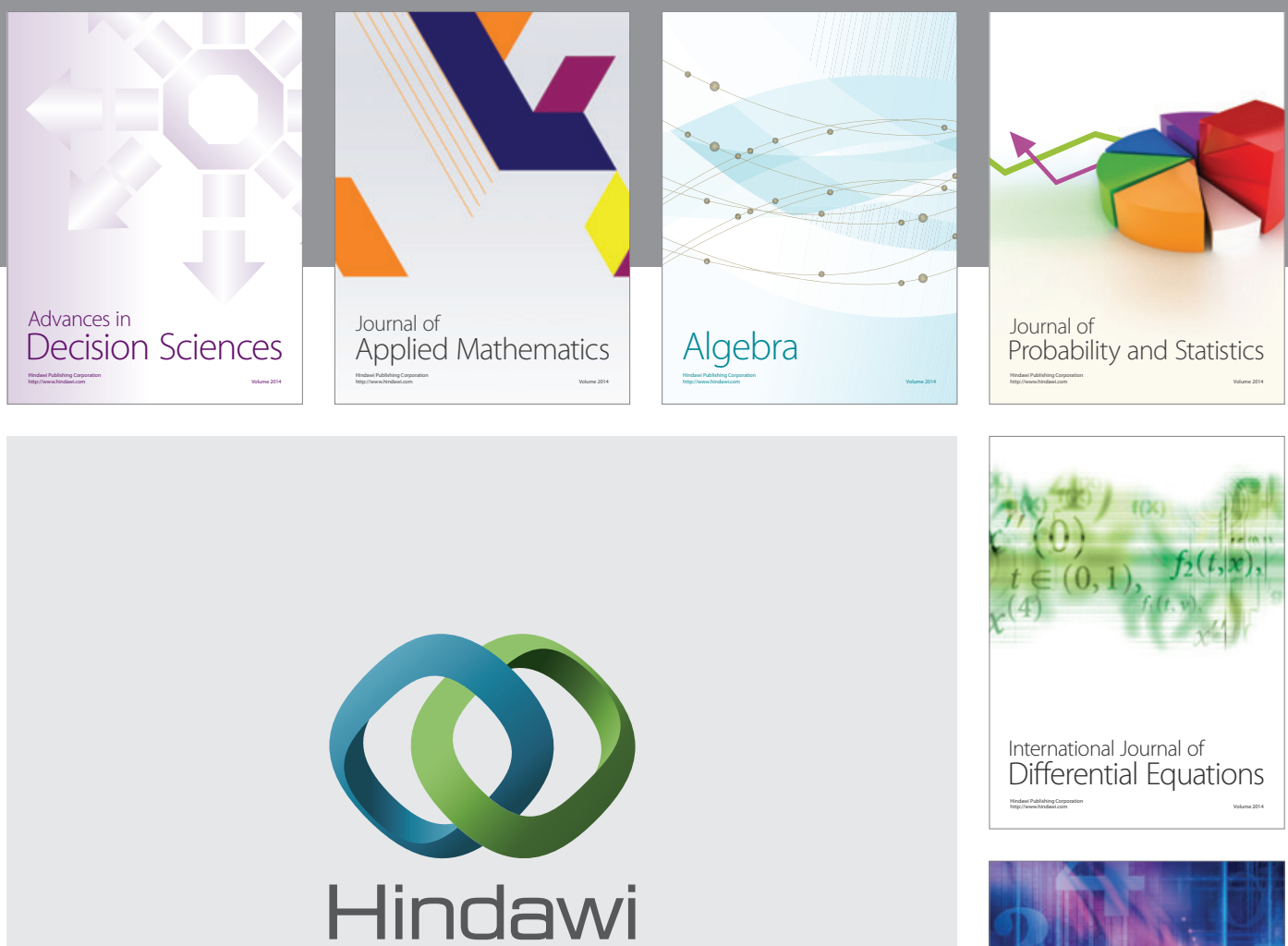

Submit your manuscripts at http://www.hindawi.com
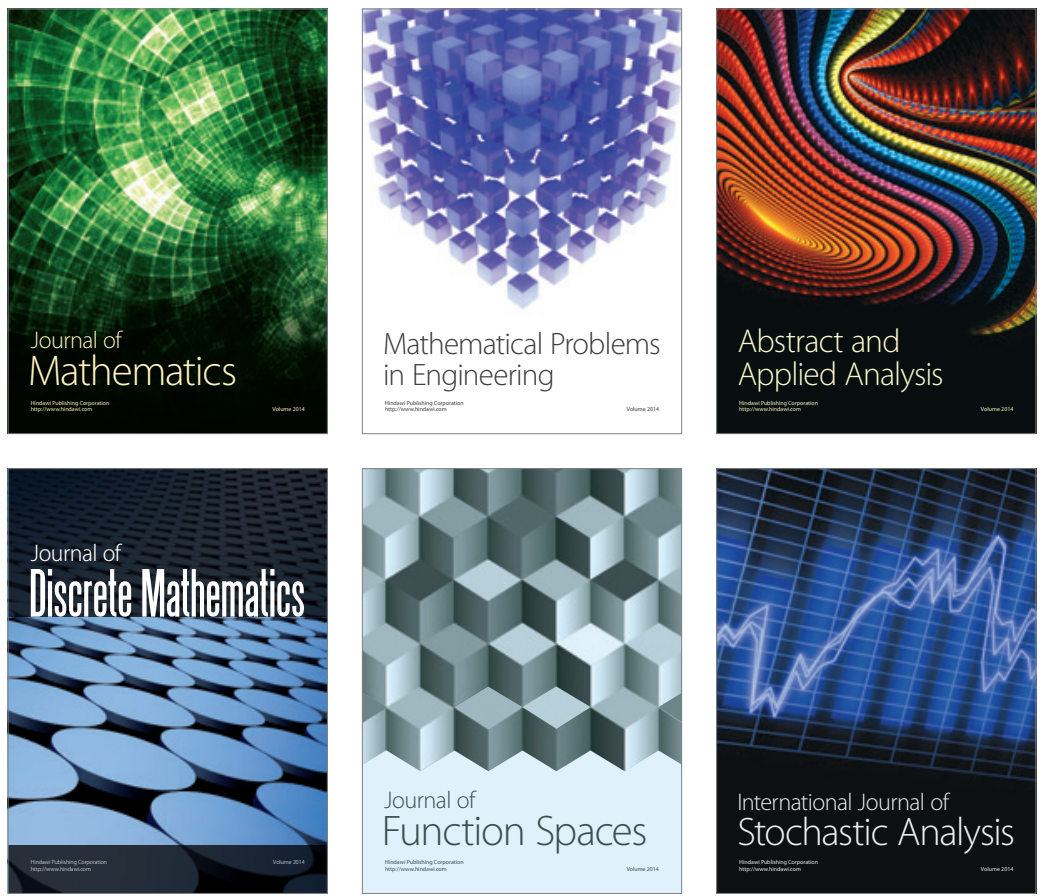

Journal of

Function Spaces

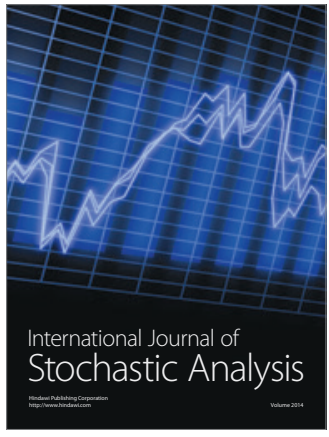

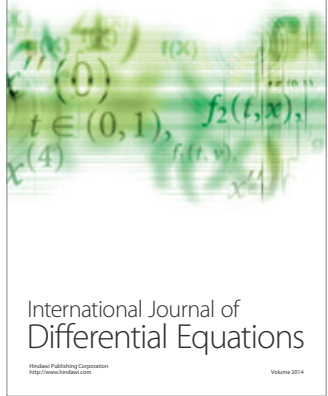
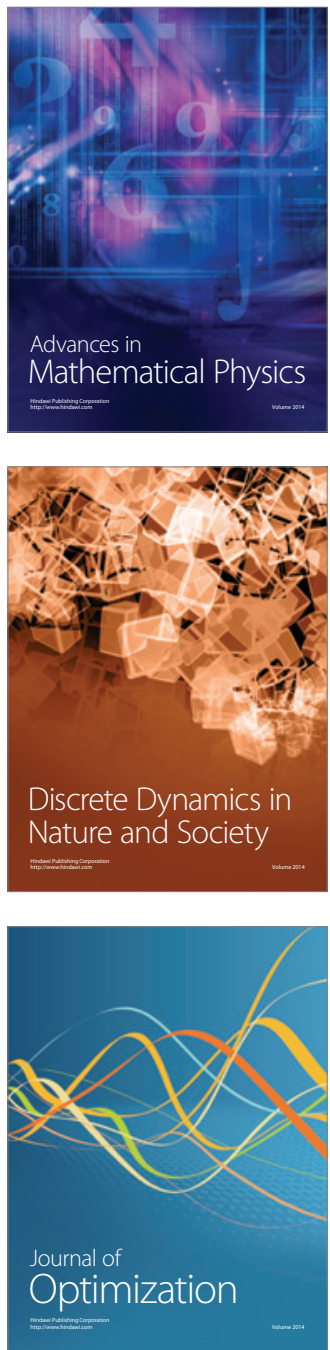